\title{
KLK1 and ZG16B proteins and arginine-proline metabolism identified as novel targets to monitor atherosclerosis, acute coronary syndrome and recovery
}

\author{
Marta Martin-Lorenzo • Irene Zubiri • Aroa S. Maroto • Laura Gonzalez-Calero • \\ Maria Posada-Ayala • Fernando de la Cuesta • Laura Mourino-Alvarez • \\ Luis F. Lopez-Almodovar • Eva Calvo-Bonacho • Luis M. Ruilope • \\ Luis R. Padial • Maria G. Barderas · Fernando Vivanco • Gloria Alvarez-Llamas
}

Received: 5 July 2014 / Accepted: 3 December 2014/Published online: 14 December 2014

(C) Springer Science+Business Media New York 2014

\begin{abstract}
We pursued here the identification of specific signatures of proteins and metabolites in urine which respond to atherosclerosis development, acute event and/or recovery. An animal model (rabbit) of atherosclerosis was developed and molecules responding to atherosclerosis silent development were identified. Those molecules were investigated in human urine from patients suffering an acute coronary syndrome (ACS), at onset and discharge. Kallikrein1 (KLK1) and zymogen granule protein16B (ZG16B) proteins, and L-alanine, L-arabitol, scyllo-inositol, 2-hydroxyphenilacetic acid, 3-hydroxybutyric acid and $\mathrm{N}$ acetylneuraminic acid metabolites were found altered in response to atherosclerosis progression and the acute event, composing a molecular panel related to cardiovascular risk. KLK1 and ZG16B together with 3-hydroxybutyric acid, putrescine and 1-methylhydantoin responded at onset but also showed normalized levels at discharge, constituting a molecular panel to monitor recovery. The observed
\end{abstract}

Electronic supplementary material The online version of this article (doi:10.1007/s11306-014-0761-8) contains supplementary material, which is available to authorized users.

M. Martin-Lorenzo · I. Zubiri · A. S. Maroto ·

L. Gonzalez-Calero - M. Posada-Ayala · F. Vivanco .

G. Alvarez-Llamas ( $\square)$

Department of Immunology, IIS-Fundacion Jimenez Diaz,

UAM, REDinREN, Avenida Reyes Católicos 2, 28040 Madrid,

Spain

e-mail: galvarez@fjd.es

F. de la Cuesta - L. Mourino-Alvarez · M. G. Barderas

Department of Vascular Physiopathology, Hospital Nacional de

Parapléjicos, SESCAM, Toledo, Spain

L. F. Lopez-Almodovar

Department of Cardiac Surgery, Hospital Virgen de la Salud,

SESCAM, Toledo, Spain decreased of KLK1 is in alignment with the protective mechanism of the kallikrein-kinin system. The connection between KLK1 and ZG16B shown by pathway analysis explains reduced levels of toll-like receptor 2 described in atherosclerosis. Metabolomic analysis revealed arginine and proline metabolism, glutathione metabolism and degradation of ketone bodies as the three main pathways altered. In conclusion, two novel urinary panels of proteins and metabolites are here for the first time shown related to atherosclerosis, ACS and patient's recovery.

Keywords Acute coronary syndrome - Urine ·

Kallikrein · Zymogen granule protein · Metabolites .

Nuclear magnetic resonance $\cdot$ MRM $\cdot$ SRM

\section{Introduction}

Cardiovascular diseases (CVD) remain the leading cause of death in developed countries and are expected to become so in emerging countries (Roger 2011; Bassand and Hamm 2007). Due to the silent nature of the process, one of the

E. Calvo-Bonacho

Ibermutuamur, Madrid, Spain

L. M. Ruilope

Cardiovascular Risk and Hypertension, Instituto de Investigacion

Hospital 12 de Octubre, Madrid, Spain

L. R. Padial

Department of Cardiology, Hospital Virgen de la Salud,

SESCAM, Toledo, Spain

F. Vivanco

Department of Biochemistry and Molecular Biology I, UCM, Madrid, Spain 
main questions is how to improve current monitoring of plaque formation, identify people at high cardiovascular risk and prevent a fatal event (Libby et al. 2011; Naghavi et al. 2003a, b). To approach molecular mechanisms underlying atherosclerosis development, we chose here an omics strategy in view of the complexity of the interactions involved and cross-talk among the different organs, cells and molecules (Corti et al. 2004; Libby and Theroux 2005). At the discovery phase, no potential marker, no key target is pre-selected, but all the proteins and/or metabolites are investigated as a whole. Thus, those molecules whose relationship with the patho-physiological processes taking place is still unknown are also investigated. In the confirmation phase, a target approach is used to specifically measure the altered molecules discovered. Previous work in our laboratory proved the potential of these omics approaches in cardiovascular research. Panels of proteins altered in plasma as a consequence of an acute coronary syndrome (ACS) (Darde et al. 2010) and proteins differentially secreted to the extracellular space from the human atherosclerotic tissue or healthy arteries (de la Cuesta et al. 2012), proving the potential of these omics approaches in cardiovascular research, in the search for markers of disease as well as in the understanding of molecular mechanisms directly occurring at tissue level (de la Cuesta et al. 2011, 2013). Similarly, different studies have pointed to panels of metabolites related to cardiovascular diseases (Barderas et al. 2011; Lewis et al. 2008; Rhee and Gerszten 2012).

We investigated the existence of a molecular panel of proteins and metabolites in urine which specifically respond to atherosclerosis in an animal model. Those proteins and metabolites identified as responders to atherosclerosis progression in animal samples were investigated, as a proof of concept, once atherosclerosis development has reached its maximum expression (an acute event) in human samples, to investigate a potential translation of main findings in animal model to human response. For such purpose, human urine was collected from patients at the onset of an ACS and at hospital discharge to specifically evaluate if those proteins and metabolites found altered in animal urine in response to atherosclerosis were also responders to the event condition itself and, if so, if they additionally normalize their levels once the patient has been discharged, i.e. return to control values.

\section{Methods}

\subsection{Animal model of atherosclerosis}

Fourteen male New Zealand White rabbits (weight $2-2.5 \mathrm{~kg}$ ) were divided in two study groups (7 animals per group): (1) animals fed with normal rabbit chow (control group); (2) animals fed with $1 \%$ cholesterol-enriched chow plus 50,000 IU/Kg vitamin D2 (Harlan, Indianapolis, Indiana) (pathological group). Vitamin D2 has been shown to accelerate the atherosclerotic process in this animal model by means of calcification (Drolet et al. 2003). The high cholesterol diet rabbit model has been widely used for experimental atherosclerosis. The observed lesions resemble, at least partially, those seen in human plaques, mainly regarding the inflammatory component. Animals were housed in individual cages in an air-conditioned room under a 12:12-h light-dark cycle. Principles of laboratory animal care were followed and all experimental procedures were approved by the Animal Care and Use Committee of the IIS-Fundación Jiménez Díaz, according to the guidelines for ethical care of the European Community. Blood samples were taken through the marginal vein of the ear at baseline, middle and end of the experimental model for the measurement of cholesterol and triglycerides by using Advia Chemistry systems (Siemens Healthcare Diagnostic Inc.). The sacrifice took place 13 weeks after. Animals were sedated with an injection of ketamine $(100 \mathrm{mg} / \mathrm{kg})$ and xylazine $(20 \mathrm{mg} / \mathrm{kg})$ and then euthanized by injection of pentobarbital $(50 \mathrm{mg} / \mathrm{kg})$ directly in the heart. Ascending aortic section was dissected, rinsed in saline buffer and embedded in paraffin. Cross-sectional serial $5 \mu \mathrm{m}$ sections were subjected to Hematoxilin-Eosin (H\&E), red alizarin staining (for visualization of calcium deposits) and immunohistochemistry (IHC) to localize macrophages and vascular smooth muscle cells (VSMCs) using antibodies against RAM11 and actin, respectively. Animals were sacrificed the same day early in the morning to minimize variation. Urine samples were taken directly from the urinary bladder at the time of sacrifice for proteins and metabolites analysis.

\subsection{Human urine samples}

Urine samples from patients were collected in sterile containers at the Cardiology Division in Hospital Virgen de la Salud (Toledo), and directly transported to the Immunology Department in IIS-Fundación Jiménez Díaz. Fourteen individuals were admitted in the study and were classified as healthy (control group) or with ACS at two time points: at hospital admission (ACSt0) and at hospital discharge (ACSt1). Urine samples from patients were taken at both time points in any case following fasting specifically, as this condition is not feasible to be guaranteed particularly at $\mathrm{t} 0$. The same was applied for control samples which were randomly taken along the morning. Sample collection procedures were in accordance with the Helsinki declaration and were approved by the institution's ethics committee. All subjects received all appropriate information 
and signed a confidentiality agreement. The control group did not receive any medication known to interfere with the studied variables. Clinical data are shown in Table 1.

\subsection{Proteomic analysis of animal urine samples} by differential gel electrophoresis (DIGE)

Animal urine samples were centrifuged at $3,000 \times g$ for 10 min to eliminate cell debris and supernatants were frozen at $-80{ }^{\circ} \mathrm{C}$ until analysis. Frozen samples were thawed in a water bath at $37^{\circ} \mathrm{C}$, filtered through Acrodisc ${ }^{\circledR}$ Syringe filters $0.2 \mu \mathrm{m}$ (Pall) and concentrated in Amicon Ultra Centrifugal Filters $10 \mathrm{kDa}$ cut-off (Millipore) up to a final volume of $300-350 \mu \mathrm{L}$. Milli $\mathrm{Q}$ water was added to a total volume of $500 \mu \mathrm{L}$ and samples clean-up was carried out by solid phase extraction with Oasis HLB cartridges (Waters, Milford, MA), following manufacturer's instructions. Solvent was removed in a Savant SpeedVac ${ }^{\circledR}$ concentrator up to dryness and samples were dissolved in $100 \mu \mathrm{L}$ lysis buffer (7 M urea, $2 \mathrm{M}$ thiourea, $4 \%$ CHAPS) and $\mathrm{pH}$ was adjusted to 8.0-8.5 with $\mathrm{NaOH}$ to perform differential gel electrophoresis (DIGE) analysis. Samples were loaded onto IPG strips (24 cm, pH 4-7). Isoelectric focusing (IEF) was carried out in a PROTEAN IEF CELL (BioRad) according to the following program: active rehydration at $50 \mathrm{~V}$ for $12 \mathrm{~h}, 500 \mathrm{~V}$ for $1 \mathrm{~h}$, up to $3,500 \mathrm{~V}$ for $3 \mathrm{~h}$ (linear voltage ramping method), $3,500 \mathrm{~V}$ for $3 \mathrm{~h}$ and up to 5,000 until 45,000 V were accumulated. Second dimension was carried out on $14 \%$ running gels using EttanDaltSix System (GE Healthcare). Gels were scanned

Table 1 Clinical data

\begin{tabular}{lllllll}
\hline Subject & Sex & Age & HTN & Diabetes & Dyslipidemia & ACS \\
\hline 1 & $\mathrm{M}$ & 57 & $\mathrm{~N}$ & $\mathrm{~N}$ & $\mathrm{~N}$ & $\mathrm{~N}$ \\
2 & $\mathrm{~F}$ & 62 & $\mathrm{Y}$ & $\mathrm{N}$ & $\mathrm{Y}$ & $\mathrm{N}$ \\
3 & $\mathrm{M}$ & 58 & $\mathrm{~N}$ & $\mathrm{~N}$ & $\mathrm{Y}$ & $\mathrm{N}$ \\
4 & $\mathrm{~F}$ & 58 & $\mathrm{~N}$ & $\mathrm{~N}$ & $\mathrm{Y}$ & $\mathrm{N}$ \\
5 & $\mathrm{M}$ & 58 & $\mathrm{~N}$ & $\mathrm{~N}$ & $\mathrm{~N}$ & $\mathrm{~N}$ \\
6 & $\mathrm{M}$ & 53 & $\mathrm{Y}$ & $\mathrm{N}$ & $\mathrm{N}$ & $\mathrm{N}$ \\
7 & $\mathrm{M}$ & 52 & $\mathrm{Y}$ & $\mathrm{N}$ & $\mathrm{Y}$ & $\mathrm{N}$ \\
8 & $\mathrm{M}$ & 80 & $\mathrm{Y}$ & $\mathrm{Y}$ & $\mathrm{Y}$ & $\mathrm{N}$ \\
9 & $\mathrm{~F}$ & 70 & $\mathrm{Y}$ & $\mathrm{N}$ & $\mathrm{N}$ & $\mathrm{N}$ \\
10 & $\mathrm{M}$ & 52 & $\mathrm{~N}$ & $\mathrm{~N}$ & $\mathrm{~N}$ & NSTEACS \\
11 & $\mathrm{M}$ & 63 & $\mathrm{Y}$ & $\mathrm{N}$ & $\mathrm{Y}$ & STEAMI \\
12 & $\mathrm{M}$ & 54 & $\mathrm{~N}$ & $\mathrm{~N}$ & $\mathrm{~N}$ & STEACS \\
13 & $\mathrm{M}$ & 73 & $\mathrm{Y}$ & $\mathrm{N}$ & $\mathrm{Y}$ & STEACS \\
14 & $\mathrm{M}$ & 65 & $\mathrm{Y}$ & $\mathrm{N}$ & $\mathrm{Y}$ & NSTEACS \\
\hline
\end{tabular}

HTN Hypertension, ACS acute coronary syndrome, AMI acute myocardial infarction, NSTEACS non-ST segment elevation of ACS. STEACS ST segment elevation of ACS. STEAMI ST segment elevation of acute myocardial infarction. Control group: subjects 1-9; ACS group: subjects $10-14$ using a Typhoon 9400 Variable Mode Imager (GE Healthcare) and spot maps were processed, analyzed and compared using the DeCyder Differential Analysis Software version 6.5 (GE Healthcare). Spot detection and normalized volume ratio calculations were performed in the Differential In-gel Analysis (DIA) module, while gelto-gel matching and statistical analysis were performed in the Biological Variation Analysis (BVA) module. Student's $t$ test was used to compare the expression of each spot. Spots found significantly varied $(p$ value $\leq 0.05)$ and with a fold change greater than 1.6 or lower than -1.6 were considered for further analysis.

\subsection{Identification of the significantly varied proteins by MALDI-TOF/TOF}

Protein spots were excised manually and automatically digested using the Ettan Digester (GE Healthcare). The digestion protocol used was that of Shevchenko et al. (1996) with minor variations. $0.5 \mu \mathrm{L}$ of each digested peptide solution were deposited using the thin layer method, onto a 384 Opti-TOF $123 \times 81 \mathrm{~mm}$ MALDI plate (Applied Biosystems) and allowed to dry at room temperature. The same volume of matrix ( $3 \mathrm{mg} / \mathrm{mL} \alpha$-cyano-4hydroxycinnamic acid (CHCA) (Sigma Aldrich) in $60 \%$ acetonitrile, $0.5 \%$ trifluoroacetic acid) was applied on every sample in the MALDI plate. Data were obtained in an automated analysis loop using a 4800 Plus MALDI TOF/TOF Analyzer (Applied Biosystems). Spectra were acquired in the reflector positive-ion mode with a Nd:YAG, $355 \mathrm{~nm}$ wavelength laser, at $200 \mathrm{~Hz}$ laser frequency, and 1,000-2,000 individual spectra were averaged. Automated analysis of mass data was performed using the 4,000 Series Explorer Software version 3.5.3 (Applied Biosystems). MALDI-MS and MS/MS data were combined through the GPS Explorer Software Version 3.6 to search a nonredundant protein database (Swissprot 2011_09) using the Mascot software version 2.2 (Matrix Science) (Perkins et al. 1999).

\subsection{Metabolomic analysis of animal urine samples by ${ }^{1} \mathrm{H}$ NMR}

Nuclear Magnetic Resonance (NMR) analysis was carried out as described previously (Posada-Ayala et al. 2013). Briefly, animal urine samples were centrifuged at $31,000 \times g$ for $15 \mathrm{~min}$ and supernatants were frozen at $-80{ }^{\circ} \mathrm{C}$ until processing. Frozen samples were thawed in a water bath at $37{ }^{\circ} \mathrm{C}$ and then $400 \mu \mathrm{L}$ sample were diluted (1:1) with $0.01 \mathrm{mM}$ sodium trimethylsilyl propionate (TSP) solution (as internal reference for spectra calibration) in $\mathrm{D}_{2} \mathrm{O}$ buffered with $\mathrm{Na}_{2} \mathrm{HPO}_{4} / \mathrm{NaH}_{2} \mathrm{PO}_{4}(200 \mathrm{mM}$, each) to $\mathrm{pH}$ 7.0. All NMR experiments were performed at 
$277 \mathrm{~K}$ on a Bruker AVANCE III 700 instrument with a $5 \mathrm{~mm}$ TCI cryoprobe equipped with shielded $\mathrm{z}$-gradient coil operating at $700.17 \mathrm{MHz}{ }^{1} \mathrm{H}$ resonance frequency. ${ }^{1} \mathrm{H}$ NMR spectra were measured with 256 scans into $32 \mathrm{~K}$ data points over a spectral width of $8,417.51 \mathrm{~Hz}$, which results in an acquisition time of $1.94 \mathrm{~s}$. A relaxation delay (d1) of $2 \mathrm{~s}$ ensured the T1-relaxation between successive scans. The signal of the solvent was suppressed by using noesyprld pulse sequence (Bruker Biospin Ltd.) in which the residual water peak is irradiated during the relaxation delay and during the mixing time of $150 \mathrm{~ms}$. All spectra were processed using TOPSPIN (version 1.3, Bruker Biospin Ltd.). Prior to Fourier transformation, the FIDs were multiplied by an exponential weight function corresponding to a line broadening of $0.3 \mathrm{~Hz}$. Spectra were phased, baselinecorrected and referenced to the TSP singlet at $\delta 0 \mathrm{ppm} .{ }^{1} \mathrm{H}$ NMR spectra were data analyzed using the software program AMIX (Analysis of MIXtures version 3.6.8, Bruker Rheinstetten, Germany). Individual integral regions were normalized to the total sum of integral region following exclusion of the water resonance. Each spectrum from 10.00 to $0 \mathrm{ppm}$ was partitioned into small spectral regions of $0.04 \mathrm{ppm}$ (buckets) thus reducing the number of total variables and compensating for small shifts in the spectra (Holmes et al. 1994) prior to statistical analysis. Nonsupervised principal components analysis (PCA) were applied to the bucket tables of spectra (no scaling). Loading plots were also calculated to show how principal component analysis is related to the original buckets.

\subsection{Metabolites identification by two dimensional NMR (2D NMR)}

For metabolites identification, spectra were analyzed by ACD/NMR Processor Academic Edition (Version 12.01, Advance Chemistry Development Inc). Unequivocal identification of metabolites was accomplished by using Metabohunter tool (Tulpan et al. 2011) and 2D NMR experimental data: homonuclear correlation spectroscopy ${ }^{1} \mathrm{H}-{ }^{1} \mathrm{H}$ (COSY), total correlation spectroscopy (TOCSY) and heteronuclear single-quantum correlation spectroscopy $\left({ }^{1} \mathrm{H}-{ }^{13} \mathrm{C}\right.$ HSQC).

\subsection{Selected reaction monitoring (SRM) analysis} of animal and human urine samples

Urine samples were analyzed in SRM mode (Selected Reaction Monitoring) (Picotti and Aebersold 2012) using a 6460 Triple Quadrupole LC-MS/MS on-line connected to: a) nano-chromatography in a Chip-format configuration (ChipCube interface, ProtID Zorbax 300B-C18-5 $\mu \mathrm{m}$ chip, Agilent Technologies) constituted by $43 \times 0.075-\mathrm{mm}$ analytical column and $40 \mathrm{~nL}$ enrichment column for proteins analysis or b) a reversed-phase column (Atlantis T3, $3 \mu \mathrm{m}, 2.1 \times 100 \mathrm{~mm}$, Waters) thermostated at $40{ }^{\circ} \mathrm{C}$ for metabolites analysis. The HPLC system consisted of a degasser, two binary pumps and thermostated autosampler maintained at $4{ }^{\circ} \mathrm{C}$ (1200 Series, Agilent Technologies). The system was controlled by Mass Hunter Software (v4.0 Agilent Technologies).

For protein analysis urine samples were concentrated in Amicon Ultra Centrifugal Filters, $10 \mathrm{kDa}$ cut-off (Millipore). Total protein content was quantified by Bradford assay. Protein samples were reduced, alkylated and digested with sequencing grade modified bovine trypsin (Roche) at a final concentration of 1:20 (trypsin:protein). Tryptic peptides solutions were cleaned with C18 spin columns (Protea Biosciences) according to manufacturer's instructions and mixed $1: 1$ with mobile phase $A(0.1 \%$ formic acid in MilliQ water). Two microliters of sample was injected at $4 \mu \mathrm{L} / \mathrm{min}$ and separation took place at $0.4 \mu \mathrm{L} /$ min in a continuous acetonitrile gradient as follows: (1) At $0 \min 5 \% \mathrm{~B}(0.1 \%$ formic acid in acetonitrile), (2) At $1 \min 5 \% \mathrm{~B}$, (3) At $5 \min 40 \% \mathrm{~B}$, (4) At $12 \min 95 \% \mathrm{~B}$, (5) At $14 \min 95 \% \mathrm{~B}$, (6) At $14.2 \min 5 \% \mathrm{~B}$ and (7) At $15 \min 5 \% \mathrm{~B}$. The mass spectrometer operated in positive mode with capillary voltage of $1,990 \mathrm{~V}, 325{ }^{\circ} \mathrm{C}$ source gas temperature and $5 \mathrm{~L} / \mathrm{min}$ source gas flow. Fragmentor was set to $130 \mathrm{~V}$, dwell time to 20 or $50 \mathrm{~ms}$, delta EMV to $600 \mathrm{~V}$ and collision energy was optimized for each SRM transition. Theoretical SRM transitions were designed using Skyline (v.1.1.0.2905) (MacLean et al. 2010) and peptide specificity was confirmed by protein blast. Only proteotypic peptides were selected.

For metabolites analysis, acetonitrile was added to urine samples (1:1) for protein precipitation and removal. Supernatants were taken, filtered through $0.20 \mu \mathrm{m}$ and diluted (1:2 or $1: 6)$ with mobile phase A $(0.1 \%$ formic acid in Milli-Q water). A sample volume of $10 \mu \mathrm{L}$ was injected and separation took place at $0.4 \mathrm{~mL} / \mathrm{min}$ in an acetonitrile gradient: (1) at $0 \mathrm{~min} 0 \% \mathrm{~B}(0.1 \%$ formic acid in acetonitrile), (2) at $0.5 \mathrm{~min} 0 \% \mathrm{~B},(3)$ at $2.5 \mathrm{~min}$ $95 \% \mathrm{~B}(4)$ at $2.51 \mathrm{~min} 0 \% \mathrm{~B}(5)$ at $3 \mathrm{~min} 0 \% \mathrm{~B}$ and a post time of $2 \mathrm{~min}$. The mass spectrometer operated in positive or negative mode with $300{ }^{\circ} \mathrm{C}$ source gas temperature and $5 \mathrm{~L} / \mathrm{min}$ source gas flow. Fragmentor potential was optimized for each metabolite in the range $60-175 \mathrm{~V}$, dwell time was fixed to $50 \mathrm{~ms}$ in positive mode and $100 \mathrm{~ms}$ in negative mode (delta EMV was fixed to 600 or $0 \mathrm{~V}$ in positive or negative mode, respectively). Collision energy was optimized for each metabolite by means of Optimizer Software (Agilent Technologies). Optimal SRM transitions were selected in direct infusion mode by previous analysis of commercial metabolite standards (see Suppl. Mat. Table 1). Individual signals were normalized based on total 
ion current (TIC) and normalized peak areas were calculated for comparison. Unpaired $t$ test was calculated by GraphPad Prism 6 (version 6.03) software.

\subsection{Pathway analysis}

Protein pathway analysis was carried out by STRING 9.0 software (Jensen et al. 2009) consisting of a database of known and predicted protein interactions, including direct (physical) and indirect (functional) associations derived from genomics, high-throughput experiments, coexpression, and previous knowledge. High confidence filter (0.600) was applied and connections involving only 2 proteins were not considered. Metabolites pathway analysis was done using MetaboAnalyst 2.0 (Xia et al. 2009, 2012) by compound matching using Homo Sapiens library, performing Fishers' exact test.

\section{Results}

\subsection{Urine molecular variations in response} to atherosclerosis silent development

This work aimed first at the identification of a molecular panel in urine associated with atherosclerosis development in an animal model. The high cholesterol diet rabbit model has been widely used for experimental atherosclerosis. The observed lesions resemble, at least partially, those seen in human plaques, mainly regarding the inflammatory component. Serum total cholesterol, LDL cholesterol, and triglycerides were measured at the beginning, middle and end of the experimental model showing a progressive increment (Suppl. Mat. Table 2). Histological characterization of aortic sections dissected from animals depicted atherosclerosis development in the special diet group (Fig. 1). In this pathological group (1-4A), compared to control animals (1-4B), intimal thickening has occurred (1A), calcium deposits are observed (2A) and migration of VSMC to the thickened intima of the aorta (3A) together with abundant macrophage infiltrate (4A) can be appreciated.

Proteins and metabolites significantly varied in urine from animals in response to atherosclerosis were investigated. PCA from proteome differential analysis by DIGE shows correct grouping of control and pathological groups and perfect separation by PC1 (Suppl. Mat. Figure 1). Proteins identification (Suppl. Mat. Table 3) revealed cathepsin D, and superoxide dismutase significantly increased in the special diet group, while hemopexin, kallikrein 1 (KLK1), and zymogen granule protein 16 homo$\log$ (ZG16B) significantly decreased. Protein variations were confirmed by Selected Reaction Monitoring (SRM)LC-MS/MS analysis in animal urine samples for cathepsin D, hemopexin, KLK1, and ZG16B (see Table 2).
Metabolomic analysis was performed by ${ }^{1} \mathrm{H}-\mathrm{NMR}$. PCA showed a good clustering for cases and controls (Suppl. Mat. Figure 2). From a total of 96 chemical shifts in the spectra showing the strongest contribution to the PCA, 37 metabolites were identified as potentially significant and analyzed by (SRM)-LC-MS/MS (see Suppl. Table 4). 19 metabolites were confirmed as significantly varied between control and pathological animal groups. Table 2 shows the different trends and significant variations. A total of 7 metabolites increased in urine in response to atherosclerosis development: L-alanine, betaine, dimethylglycine, glycine, taurine, L-arabitol and 1-methylhydantoin. Twelve metabolites show the opposite trend (decreased in response to atherosclerosis): p-benzoquinone, pipecolic acid, L-serine, putrescine, 6-phosphogluconic acid, cyclohexanol, pyrocathecol, hypotaurine, L-lysine, spermidine, tyramine and scyllo-inositol.

\subsection{Proteins and metabolites fingerprint in response} to ACS and recovery

As detailed above, we initially defined a molecular panel which clearly responds to atherosclerosis pathology in animals (silently and asymptomatic). In a further step and as a proof of concept of animal data transference to humans, we investigated a potential alteration of those molecules in response to the ultimate fatal consequence of atherosclerosis development, i.e. an acute event. With this aim, those molecules were further analyzed in human urine samples from ACS patients. Human urine samples were collected both at the onset of ACS and at discharge, to elucidate changes in response to the acute syndrome but also related to patient's recovery. A detailed view of the workflow followed in the study is shown in Fig. 2.

We quantitatively analyzed those proteins and metabolites which had been selected as responders to atherosclerosis in animal urine, in urine samples collected from individuals who had suffered ACS, both at admission ( $\mathrm{t} 0$ ) and at discharge (t1) time points, and in comparison with healthy individuals' urine (control group). (SRM)-LC-MS/ MS revealed different trends, providing with particular sub-sets of molecules which may serve as indicators of the acute event itself or recovery.

In particular, KLK1 and ZG16B were found significantly decreased in ACSt0 (onset), returning to control values at ACSt1 (discharge) and showing the same trend observed in the animal model (decreased in special diet group) (see Fig. 3). Although without significant variation, trends observed in the animal model were also confirmed for cathepsin D and hemopexin in human urine samples collected at ACSt0 time point compared to control levels, showing partial normalization at ACSt1.

At metabolome level, L-alanine and L-arabitol were found significantly increased, and scyllo-inositol significantly 

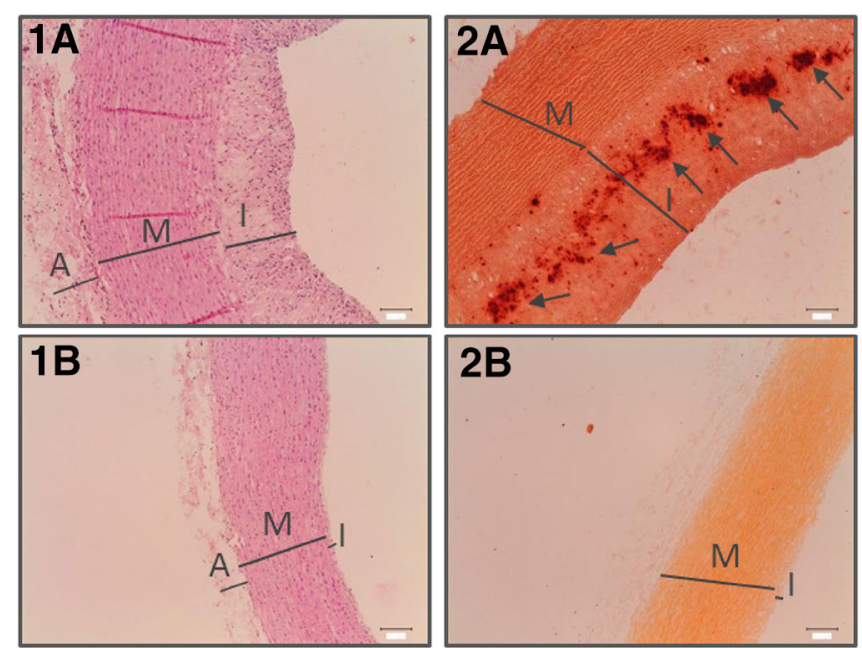

Fig. 1 Histological characterization of aortic tissue from a pathological animals (special diet) and b control animals. 1 Hematoxilin-Eosin (H\&E), 2 red alizarin staining, 3 actin and 4 RAM 11. Representative

Table 2 SRM confirmation analysis

\begin{tabular}{lll}
\hline Name & Pathological/control & Significance (p-value) \\
\hline Proteins & & \\
Cathepsin D & $\uparrow$ & $*$ \\
Hemopexin & $\downarrow$ & $*$ \\
KLK1 & $\downarrow$ & $* *$ \\
ZG16B & $\downarrow$ & $*$ \\
Metabolites & & $*$ \\
1-Methylhydantoin & $\uparrow$ & $* * *$ \\
6-Phosphogluconic & $\downarrow$ & $* * * *$ \\
Betaine & $\uparrow$ & $*$ \\
Cyclohexanol & $\downarrow$ & $* * *$ \\
Dimethylglycine & $\uparrow$ & $*$ \\
Glycine & $\uparrow$ & $* * * *$ \\
Hypotaurine & $\downarrow$ & $* *$ \\
L-Alanine & $\uparrow$ & $* *$ \\
L-Arabitol & $\uparrow$ & $* *$ \\
L-Lysine & $\downarrow$ & $* * *$ \\
L-Serine & $\downarrow$ & $* * *$ \\
p-Benzoquinone & $\downarrow$ & $* * * *$ \\
Pipecolic acid & $\downarrow$ & $*$ \\
Putrescine & $\downarrow$ & $* * * *$ \\
Pyrocatechol & $\downarrow$ & $* * * *$ \\
Scyllo-inositol & $\downarrow$ & $* * *$ \\
Spermidine & $\downarrow$ & $\uparrow *$ \\
Taurine & $\uparrow$ & $\downarrow$ \\
Tyramine & $\downarrow$ & $\downarrow$ \\
\hline
\end{tabular}

Proteins and metabolites found significantly altered in urine from pathological animals (atherosclerosis) compared to control animal group (7 animals per group).

$* p \quad$ value $<0.05, * * p \quad$ value $<0.01$, *** $p$ value $<0.001$, **** $p$ value $<0.0001$
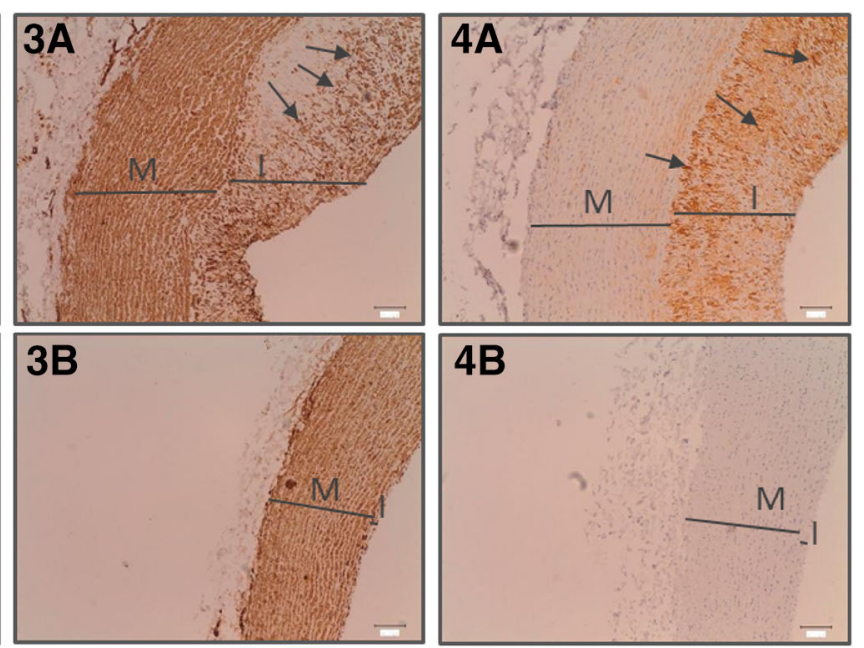

images ( $\times 10$ magnification, $100 \mu \mathrm{m}$ scale). Letter code, $A$ adventitia, $M$ media, $I$ intima. Arrows correspond to calcium deposits, SMCs and macrophages-foam cells on pictures $\mathbf{2 a}, \mathbf{3 a}$ and $\mathbf{4 a}$, respectively

decreased at ACS, both at t0 and t1, compared to control levels, showing the same trend observed in the animal model (increased or decreased in the special diet group, respectively). With opposite trend to that observed in the animal model, putrescine, spermidine, p-benzoquinone and cyclohexanol were found increased at ACSt0 versus control. Putrescine and cyclohexanol additionally showed total or partial recovery to normal values at ACSt1. 1-methylhydantoin significantly decreased at the onset (ACSt0), while normal levels are already reached at discharge (ACSt1). 2-hydroxyphenylacetic acid decreased at ACSt0, while $\mathrm{N}$-acetylneuraminic acid and 3-hydroxybutyric acid increased. Interestingly, all these three metabolites showed significant variation in response to ACS, following the same trend to that observed in the animal model, although no significant differences have been found in animals' urine. Among the three, 3-hydroxybutyric acid showed normalized levels at discharge (see Fig. 4).

Metabolic pathway analysis was performed by including metabolites found significantly altered during atherosclerosis progression (animal model) or in response to an ACS (human). Common altered pathways to both situations ( $p$ value $<0.05$ ) were "arginine and proline metabolism" (involving putrescine, spermidine and 1-methylhydantoin), and "glutathione metabolism" (including putrescine, spermidine and glycine) (Fig. 5).

\section{Discussion}

A significant alteration of the urine proteome and metabolome in response to atherosclerosis is demonstrated here. 


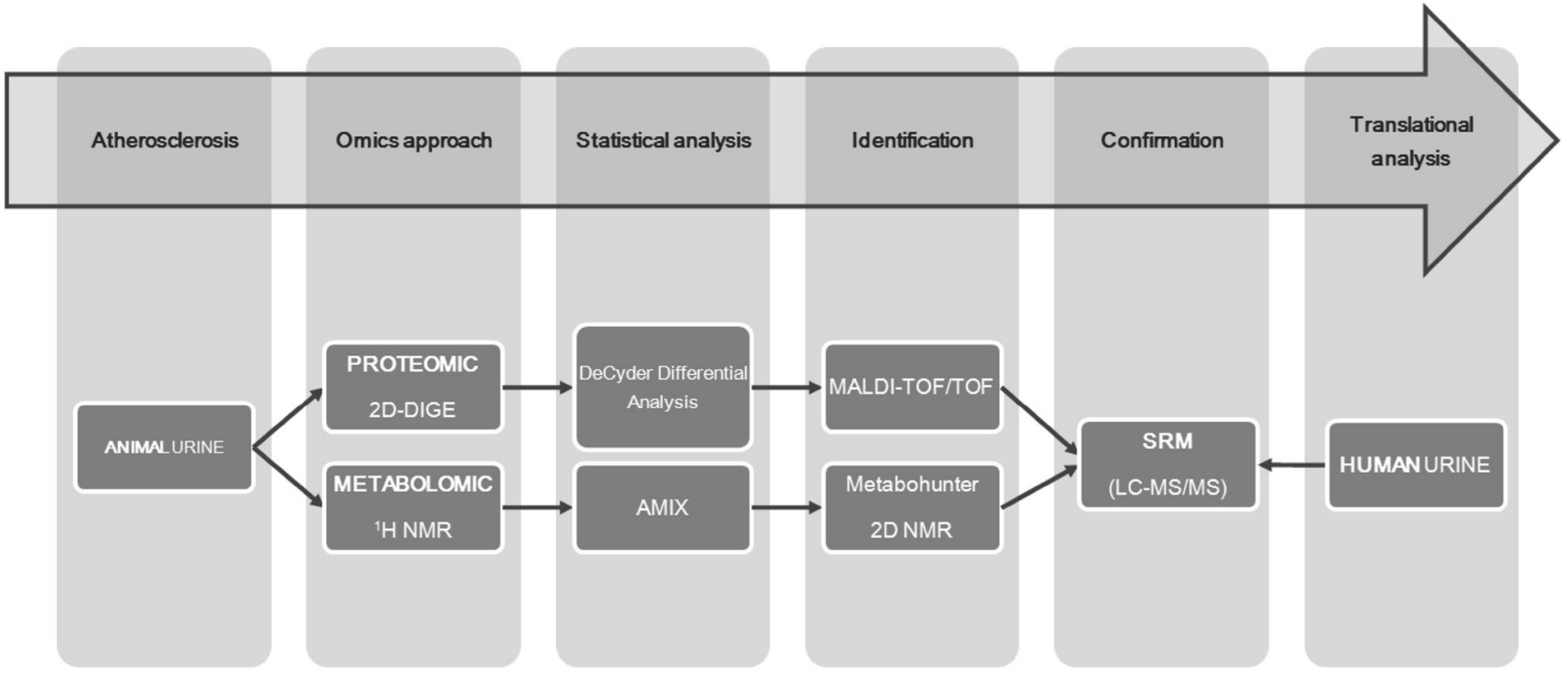

Fig. 2 Workflow schema for proteomic and metabolomic analysis of urine. Candidate markers of atherosclerosis were discovered in an animal model and further confirmed by SRM. Translation into a human fingerprint was investigated in response to ACS

Kallikrein 458.6-> 471.7

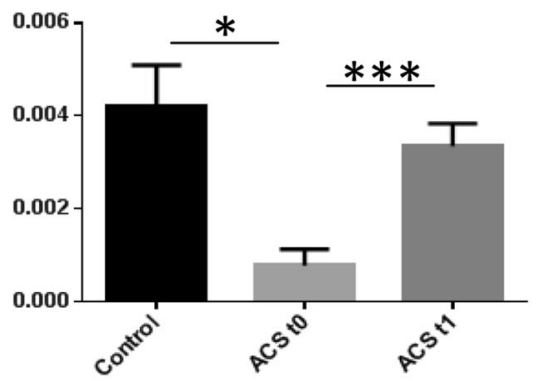

ZG16B 458.7->629.3

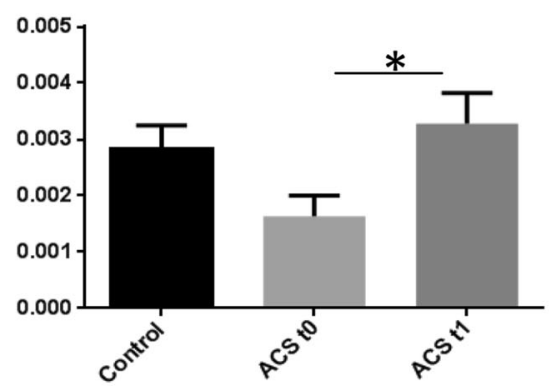

Kallikrein $458.6-529.3$

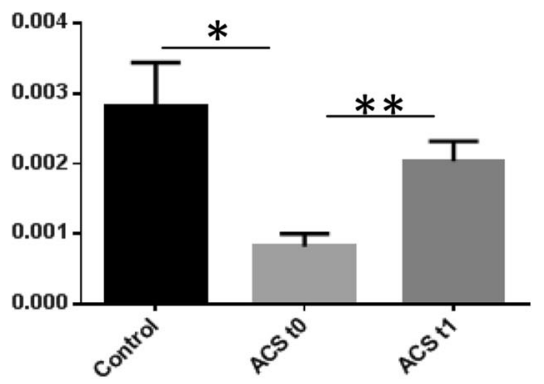

ZG16B 458.7 $>683.3$

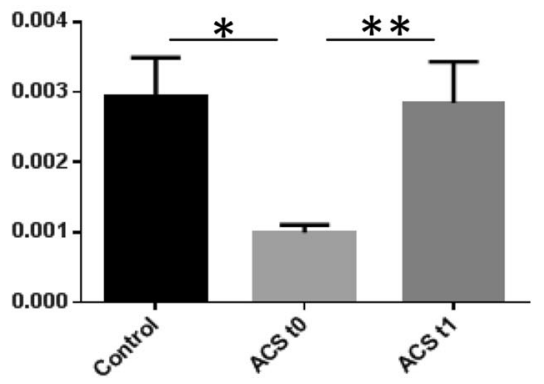

ZG16B 458.7-> 728.4

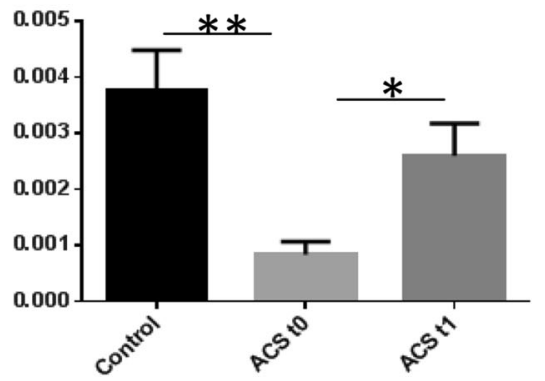

Fig. 3 SRM data showing proteins significantly altered in urine from ACS patients. Urine samples were collected from healthy subjects (control group) $(\mathrm{n}=9)$ and patients at the onset $(\mathrm{t} 0)$ and at discharge $(\mathrm{t} 1)(\mathrm{n}=5)$. Error bars represent SEM

To investigate which of those molecules responding to atherosclerosis development in the animal model correlate well with operating mechanisms in humans, we collected urine samples from individuals suffering from an acute event (ACS). This is the extreme situation and the latest effect of disease progression. Additionally, samples were collected at hospital discharge too, looking for a potential value of these markers as indicators of patients' recovery.

A sub-set of molecules showed significant variation in human urine in response to ACS and some of them returned to control values at patient discharge. A group of them responded with the same trend both to atherosclerosis 

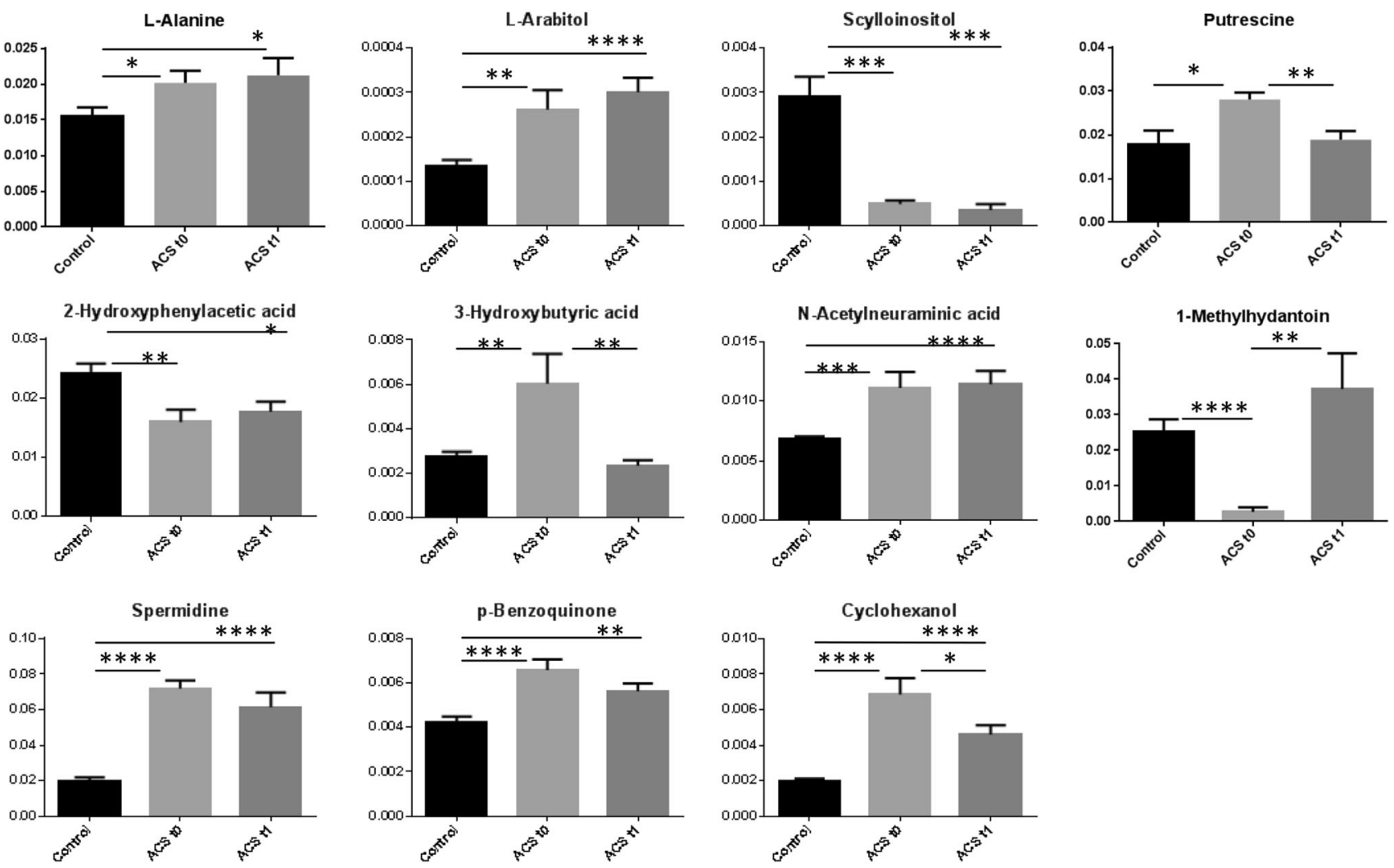

Fig. 4 SRM data showing metabolites significantly altered in urine from ACS patients. Urine samples were collected from healthy subjects (control group) $(\mathrm{n}=9)$ and patients at the onset $(\mathrm{t} 0)$ and at discharge $(\mathrm{t} 1)(\mathrm{n}=5)$. Error bars represent SEM

development (animal model) and to the acute onset (in human). That is the case for proteins KLK1 and ZG16B and metabolites L-alanine, L-arabitol, scyllo-inositol, 2-hydroxyphenylacetic acid, 3-hydroxybutyric acid and $\mathrm{N}$-acetylneuraminic acid. This panel allows monitoring disease progression and may constitute a potential signature to evaluate individual cardiovascular risk, pending further research in additional patient cohorts.

In other cases, as for metabolites putrescine, spermidine, p-benzoquinone, cyclohexanol and 1-methylhydantoin, opposite trends (increase or decrease) were observed in response to atherosclerosis development (before an acute event occurs, animal model) and to the event itself. This observation points to a mixed response in opposite directions, i.e. different mechanisms are activated in particular conditions or particular stage of the disease. In this case, particular metabolites showed altered response following a certain trend during atherosclerosis development (still asymptomatic) but this alteration is in the opposite trend when an acute syndrome takes place, indicating overlapping responses.

In relation to recovery or follow-up, a panel composed by three metabolites, 3-hydroxybutyric acid, putrescine, 1-methylhydantoin and two proteins KLK1 and ZG16B clearly responds to an ACS event and additionally shows the capability to monitor recovery, as the urinary levels returned to control values at patient's hospital discharge. Figure 6 summarizes the most relevant findings of the study.

KLK-1 is a serine protease acting in the release of the vasoactive peptide, Lys-bradykinin, from low molecular weight kininogen. These kinins are inflammatory mediators (Brain and Williams 1985). Kallikrein-kinin system is involved in the control of vascular smooth muscle tone and arterial blood pressure (Marcondes and Antunes 2005), playing a significant cardioprotective effect mostly endothelium-mediated (Dendorfer et al. 1999). It has been suggested (Sharma and Sharma 2002) that reduced activity of the local kallikrein-kinin system may be instrumental in the induction of cardiovascular-related diseases. Tissue kallikrein measurement has been shown to be useful in diagnosis and monitoring of atherosclerosis (Porcu et al. 2004) and it has been proved that tissue kallikrein infusion improves cardiac function and protect against myocardial ischemia injury after coronary artery occlusion (Yao et al. 2007). The protector mechanism is related with an inhibition of apoptosis, inflammation, hypertrophy and fibrosis, as well as an enhancement of neovascularization (Chao 


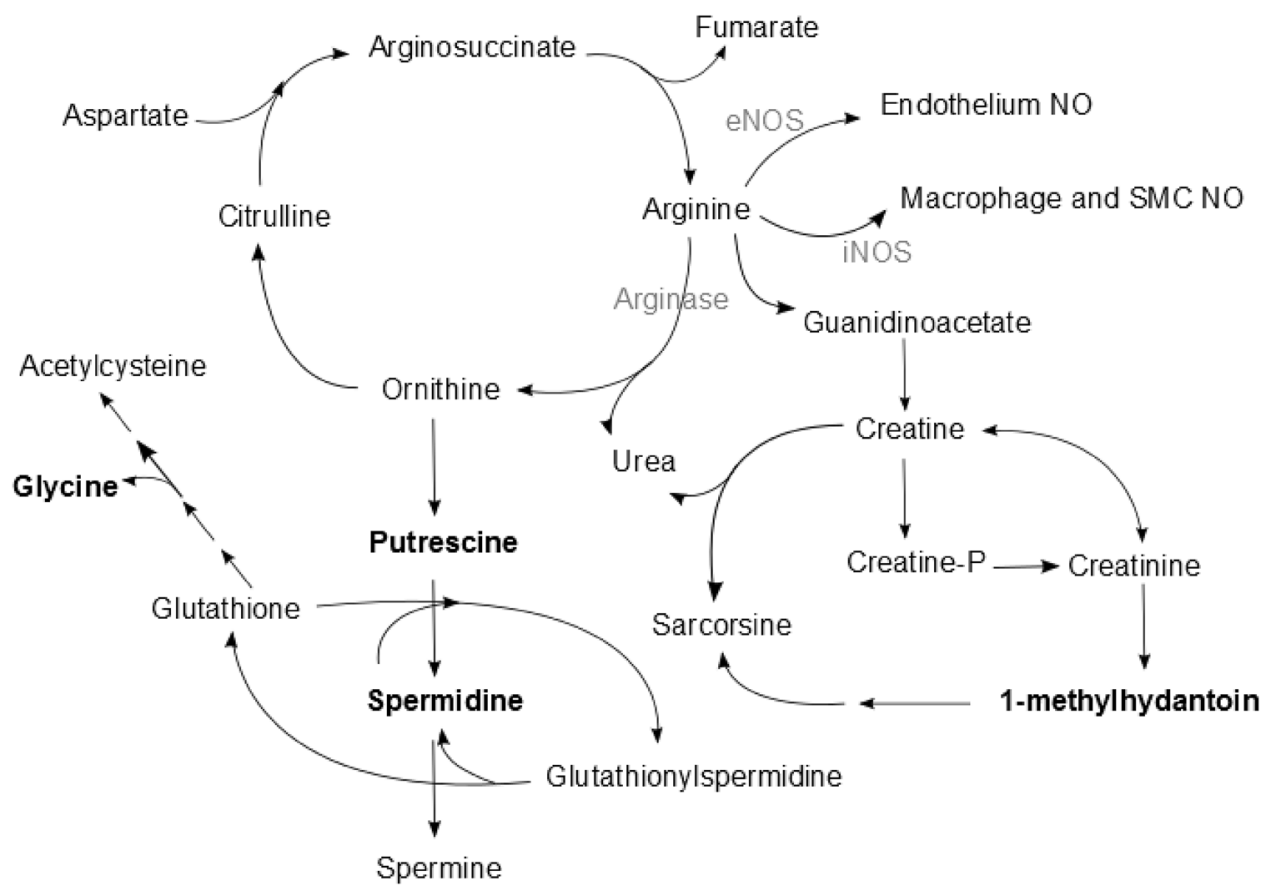

Fig. 5 Overview of key altered metabolites found in animal model (atherosclerosis development) and human urine (acute event). Main pathways involved are glutathione and arginine-proline metabolism. This map was created from KEGG pathways database, highlighting only those which are relevant in the context of metabolites found altered in this study. Role of arginine as NO precursor is also included. Metabolites found significantly altered in this study are marked in black bold letters. Enzymes are in grey. Metabolites in glutathione pathway: acetylcysteine, glycine, glutathione, spermidine and glutathionylspermidine. Metabolites in arginine-proline pathway: aspartate, arginosuccinate, fumarate, arginine, ornithine, putrescine, spermidine, spermine, guanidinoacetate, creatine, creatinine, cretaineP, 1-methylhydantoin, sarcorsine
Fig. 6 Panels of discovered metabolites and proteins as potential markers of disease progression, acute onset and/or recovery

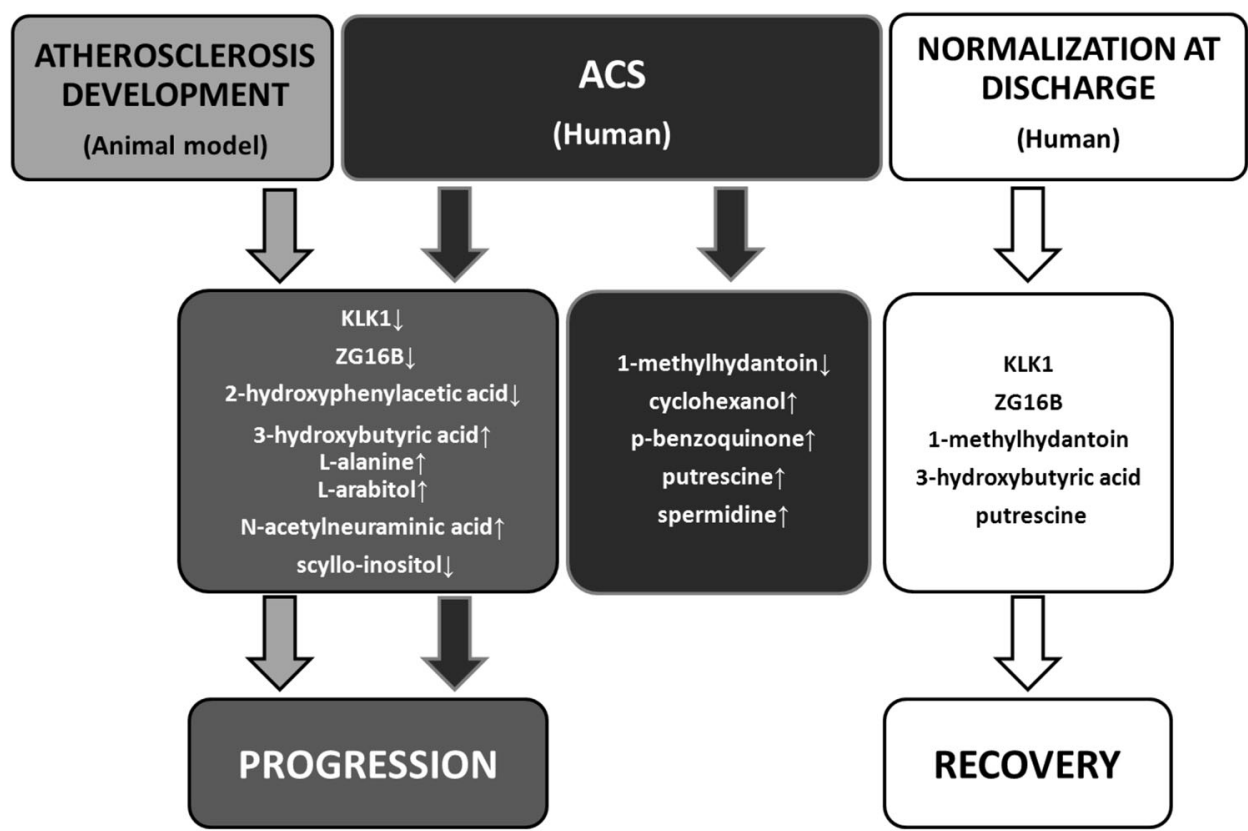

et al. 2010). These observations correlate with the observed decrease in urine for kallikrein-1 in response to atherosclerosis development and acute event (ACS), following normalization at patient's hospital discharge. The same behavior was observed for ZG16B. It has been described that ZG16B activate the chemokine CXCR4 (Lee et al. 
2010), which is a receptor of CXCL12. Levels of CXCL12 were found diminished in plasma from patients with stable and unstable angina (Damas et al. 2002). In agreement, our study shows a decrease in ZG16B urinary levels, which may imply a reduced activation of CXCL12 receptor, CXCR4.

ZG16B and KLK-1 proteins are connected by toll like receptor 2 (TLR2); KLK1 via Kininogen and ZG16B via CXCR4 (suppl. Mat. Figure 3). TLR2 has been widely related with atherosclerosis playing different and controversial roles (Mullick et al. 2005). From one side it has been described to have a beneficial effect by preserving endothelial cell function during vascular inflammatory diseases (Wagner et al. 2013). From the other side, overactivation of the endothelium by excessive TLR2 activation may promote endothelial dysfunction. We observed here a decrease of $\mathrm{ZB} 16 \mathrm{~B}$ and KLK-1 levels in the context of atherosclerosis. In view of the controversial roles attributed to TLR2 in the literature (beneficial or detrimental), reduced levels of KLK1 and ZG16B found in this study in atherosclerosis are in better consonance with a potential diminution of TLR2 levels (Supplementary Fig. 3), and thus more in agreement with a beneficial effect.

At the metabolome level, cardiovascular risk assessment of population from different geographical areas has been related to an increase in urinary L-alanine, and $\mathrm{N}$-acetyl neuraminic acid, and to a decrease in urinary scyllo-inositol, in agreement with our findings (Yap et al. 2010). Plasma 3-hydroxybutyrate was found increased in stable carotid atherosclerosis patients, and in plasma from ACS patients, in accordance with our data in human urine where this metabolite was found increased at the ACS onset and normalized at discharge (Laborde et al. 2014; Teul et al. 2009). Spermidine and putrescine have an antagonist action in platelet aggregation which explains the increase observed in this study in ACSt0 as a compensatory mechanism (de la Pena et al. 2000, Zhang et al. 2009). 1-methylhydantoin is a product of the creatinine metabolism (Wyss and Kaddurah-Daouk 2000) which has been widely described to increase in atherosclerosis (Gentile et al. 2013; Rein et al. 2014). We found this trend in atherosclerotic animals compared to the control group, but urinary levels in ACS conditions were found lower. Oxidative stress is a causative factor in this pathology and, particularly, plasma levels of oxidative stress indicators (isoprostanes) were found particularly increased in ACS, compared with stable coronary artery disease (CAD) and correlating with platelet activation (Szuldrzynski et al. 2010). In mammals, the metabolism of 1-methylhydantoin occurs via 5-hydroxymethylhydantoin, which is an intrinsic antioxidant against cellular damage (Ienaga et al. 2013). The observed decrease of urinary 1-methylhydantoin in response to an ACS correlates well with an activated metabolism in favor of 5-hydroxymethylhydantoin in response to increased levels of ROS produced by activated platelets. In vitro analysis have shown inhibition of Fc receptor- mediated phagocytosis in macrophages by benzoquinone (Manning et al. 1994). Deficiency of Fcgamma receptor has been pointed to a protective mechanism against atherosclerosis (Hernandez-Vargas et al. 2006). In our study, benzoquinone was found decreased in atherosclerosis development but increased during ACS. This observation might point to a specific activation of this protective mechanism in extreme situations, although further research should be performed.

Metabolic pathway analysis was performed by including found metabolites significantly altered during atherosclerosis progression (animal model) or in response to an ACS (human). The common pathways were arginine and proline and glutathione metabolism. Figure 5 depicts a combined view of those KEGG pathways involved. The polyamines putrescine and spermidine are produced from ornithine. They inhibit platelet aggregation and their increment is closely related with arterial injury (Durante et al. 1997) as they contribute to arterial remodelling at sites of vascular damage (Durante et al. 2001). In particular, there are two competing mechanisms for arginine between arginase and NOS (nitric oxide synthase) in favour of NO (nitric oxide) or ornithine production (precursor of polyamines and proline), respectively (Getz and Reardon 2006). An increase in polyamine synthesis in tissue has been described in response to proliferation of VSMCs and development of intimal thickening (Nishida et al. 1990). In atherosclerosis and arterial injury, arginase activity is increased showing different roles for arginase I and II in plaque development and vulnerability (Durante 2013). The increase of arginase explains the synthesis of polyamines from ornithine and in consonance with previously reported elevation of polyamines levels in tissue. We found putrescine and spermidine decreased in urine during atherosclerosis development (animal model) in agreement with the expected accumulation in tissue. Once the acute event takes place, increased polyamines levels were found in urine from ACS patients which may be a consequence of a release of the intracellular content to the blood stream and ultimately filtrated into urine, or of an activation of the polyamines inhibitory response to platelet aggregation which takes place after an acute event occurs, or both. Taking part into glutathione metabolism, $N$-acetylcysteine (also called mercapturic acid) has antioxidant and anti-inflammatory properties and promotes plaque stabilization ( $\mathrm{Lu}$ et al. 2011). It is an end product of four consecutive variations of glutathione. In the third step glycine is released, in agreement with our data showing increased levels in urine of atherosclerotic animals. 


\section{Concluding remarks}

This pilot study shows specific molecular panels associated with atherosclerosis, acute event and recovery in a noninvasive and easily accessible fluid used in routine clinical practice as urine.

Further investigation related to plaque development and instability, and evaluation of molecular panels identified here in patients without reported acute events but high cardiovascular risk, would help in defining specific signatures for disease progression and risk stratification and would cover a gap between atherosclerosis development and irreversible acute damage.

Acknowledgments Instituto de Salud Carlos III (FIS PI080970, FIS PI11/01401, FIS PI13/01873, FIS PI11/02239, FIS IF08/3667-1, FIS PS09/00447, CP09/00229, RD12/0013/0013, RD12/0042/0071, PTI3/ 0001/0013, PIE13/00051), IDCSalud (3371/002), Fondos FEDER, Fundación Conchita Rábago de Jiménez Díaz. Authors thank personnel from the Cardiology Service in Hospital Virgen de la Salud (Toledo), Proteomics Facility UCM-PCM (a member of ProteoRedISCIII network), Unidad de Proteómica Hospital Nacional de Parapléjicos (Toledo) and CAI-RMN (UCM). These results are lined up with the Spanish initiative on the Human Proteome Project (SpHPP).

Disclosure The authors declare no financial benefit from this work.

Compliance with ethical requirements All procedures performed in studies involving human participants were in accordance with the ethical standards of the institutional and/or national research committee and with the 1964 Helsinki declaration and its later amendments or comparable ethical standards. All applicable international, national, and/or institutional guidelines for the care and use of animals were followed.

\section{References}

Barderas, M. G., et al. (2011). Metabolomic profiling for identification of novel potential biomarkers in cardiovascular diseases. Journal of Biomedicine and Biotechnology, 2011, 790132. doi:10.1155/2011/790132.

Bassand, J. P., \& Hamm, C. (2007). New European guidelines for the management of patients with unstable angina/non-ST-elevation myocardial infarction-what are the new and key messages. Polskie Archiwum Medycyny Wewnetrznej, 117, 391-393.

Brain, S. D., \& Williams, T. J. (1985). Inflammatory oedema induced by synergism between calcitonin gene-related peptide (CGRP) and mediators of increased vascular permeability. British Journal of Pharmacology, 86, 855-860.

Chao, J., Shen, B., Gao, L., Xia, C. F., Bledsoe, G., \& Chao, L. (2010). Tissue kallikrein in cardiovascular, cerebrovascular and renal diseases and skin wound healing. Biological Chemistry, 391, 345-355. doi:10.1515/BC.2010.042.

Corti, R., Hutter, R., Badimon, J. J., \& Fuster, V. (2004). Evolving concepts in the triad of atherosclerosis, inflammation and thrombosis. Journal of Thrombosis and Thrombolysis, 17, $35-44$.

Damas, J. K., et al. (2002). Stromal cell-derived factor-1alpha in unstable angina: Potential antiinflammatory and matrix-stabilizing effects. Circulation, 106, 36-42.
Darde, V. M., de la Cuesta, F., Dones, F. G., Alvarez-Llamas, G., Barderas, M. G., \& Vivanco, F. (2010). Analysis of the plasma proteome associated with acute coronary syndrome: Does a permanent protein signature exist in the plasma of ACS patients? Journal of Proteome Research, 9, 4420-4432. doi:10.1021/ pr1002017.

de la Cuesta, F., et al. (2011). A proteomic focus on the alterations occurring at the human atherosclerotic coronary intima. Molecular and Cellular Proteomics, 10, M110.

de la Cuesta, F., et al. (2012). Secretome analysis of atherosclerotic and non-atherosclerotic arteries reveals dynamic extracellular remodeling during pathogenesis. Journal of Proteomics, 75, 2960-2971. doi:10.1016/j.jprot.2011.12.005.

de la Cuesta, F., et al. (2013). Deregulation of smooth muscle cell cytoskeleton within the human atherosclerotic coronary media layer. Journal of Proteomics, 82, 155-165.

de la Pena, N. C., Sosa-Melgarejo, J. A., Ramos, R. R., \& Mendez, J. D. (2000). Inhibition of platelet aggregation by putrescine, spermidine, and spermine in hypercholesterolemic rabbits. Archives of Medical Research, 31, 546-550.

Dendorfer, A., Wolfrum, S., \& Dominiak, P. (1999). Pharmacology and cardiovascular implications of the kinin-kallikrein system. Japanese Journal of Pharmacology, 79, 403-426.

Drolet, M. C., Arsenault, M., \& Couet, J. (2003). Experimental aortic valve stenosis in rabbits. Journal of the American College of Cardiology, 41, 1211-1217.

Durante, W. (2013). Role of arginase in vessel wall remodeling. Frontiers in Immunology, 4, 111. doi:10.3389/fimmu.2013. 00111.

Durante, W., Liao, L., Peyton, K. J., \& Schafer, A. I. (1997). Lysophosphatidylcholine regulates cationic amino acid transport and metabolism in vascular smooth muscle cells. Role in polyamine biosynthesis. Journal of Biological Chemistry, 272, 30154-30159.

Durante, W., Liao, L., Reyna, S. V., Peyton, K. J., \& Schafer, A. I. (2001). Transforming growth factor-beta(1) stimulates L-arginine transport and metabolism in vascular smooth muscle cells: Role in polyamine and collagen synthesis. Circulation, 103, 1121-1127.

Gentile, M., et al. (2013). Plasma creatinine levels, estimated glomerular filtration rate and carotid intima media thickness in middle-aged women: A population based cohort study. Nutrition, Metabolism and Cardiovascular Diseases, doi:10.1016/j. numecd.2013.11.012.

Getz, G. S., \& Reardon, C. A. (2006). Arginine/arginase NO NO NO. Arteriosclerosis, Thrombosis, and Vascular Biology, 26, 237-239. doi:10.1161/01.ATV.0000202014.54609.9d.

Hernandez-Vargas, P., et al. (2006). Fcgamma receptor deficiency confers protection against atherosclerosis in apolipoprotein $\mathrm{E}$ knockout mice. Circulation Research, 99, 1188-1196. doi:10. 1161/01.RES.0000250556.07796.6c.

Holmes, E., et al. (1994). Automatic data reduction and pattern recognition methods for analysis of $1 \mathrm{H}$ nuclear magnetic resonance spectra of human urine from normal and pathological states. Analytical Biochemistry, 220, 284-296.

Ienaga, K., Park, C. H., \& Yokozawa, T. (2013). Protective effect of an intrinsic antioxidant, HMH (5-hydroxy-1-methylhydantoin; NZ-419), against cellular damage of kidney tubules. Experimental and Toxicologic Pathology, 65, 559-566. doi:10.1016/j. etp.2012.05.001.

Jensen, L. J., et al. (2009). STRING 8-a global view on proteins and their functional interactions in 630 organisms. Nucleic Acids Research, 37, D412-D416. doi:10.1093/nar/gkn760.

Laborde, M., C., et al. (2014). Plasma metabolomics reveals a potential panel of biomarkers for early diagnosis in acute coronary syndrome. Metabolomics, 10, 414-424. doi:10.1007/ s11306-013-0595-9. 
Lee, Y., et al. (2010). PAUF functions in the metastasis of human pancreatic cancer cells and upregulates CXCR4 expression. Oncogene, 29, 56-67. doi:10.1038/onc.2009.298.

Lewis, G. D., Asnani, A., \& Gerszten, R. E. (2008). Application of metabolomics to cardiovascular biomarker and pathway discovery. Journal of the American College of Cardiology, 52, 117-123. doi:10.1016/j.jacc.2008.03.043.

Libby, P., Ridker, P. M., \& Hansson, G. K. (2011). Progress and challenges in translating the biology of atherosclerosis. Nature, 473, 317-325. doi:10.1038/nature10146.

Libby, P., \& Theroux, P. (2005). Pathophysiology of coronary artery disease. Circulation, 111, 3481-3488.

Lu, Y., et al. (2011). The antioxidant $\mathrm{N}$-acetylcysteine promotes atherosclerotic plaque stabilization through suppression of RAGE, MMPs and NF-kappaB in ApoE-deficient mice. Journal of Atherosclerosis and Thrombosis, 18, 998-1008.

MacLean, B., et al. (2010). Skyline: An open source document editor for creating and analyzing targeted proteomics experiments. Bioinformatics, 26, 966-968. doi:10.1093/bioinformatics/ btq054.

Manning, B. W., Adams, D. O., \& Lewis, J. G. (1994). Effects of benzene metabolites on receptor-mediated phagocytosis and cytoskeletal integrity in mouse peritoneal macrophages. Toxicology and Applied Pharmacology, 126, 214-223. doi:10.1006/ taap.1994.1110.

Marcondes, S., \& Antunes, E. (2005). The plasma and tissue kininogen-kallikrein-kinin system: Role in the cardiovascular system. Current Medicinal Chemistry: Cardiovascular \& Hematological Agents, 3, 33-44.

Mullick, A. E., Tobias, P. S., \& Curtiss, L. K. (2005). Modulation of atherosclerosis in mice by Toll-like receptor 2. Journal of Clinical Investigation, 115, 3149-3156. doi:10.1172/JCI25482.

Naghavi, M., et al. (2003a). From vulnerable plaque to vulnerable patient: A call for new definitions and risk assessment strategies: Part II. Circulation, 108, 1772-1778. doi:10.1161/01.CIR. 0000087481.55887.C9108/15/1772.

Naghavi, M., et al. (2003b). From vulnerable plaque to vulnerable patient: A call for new definitions and risk assessment strategies: Part I. Circulation, 108, 1664-1672. doi:10.1161/01.CIR. 0000087480.94275.97108/14/1664.

Nishida, K., Abiko, T., Ishihara, M., \& Tomikawa, M. (1990). Arterial injury-induced smooth muscle cell proliferation in rats is accompanied by increase in polyamine synthesis and level. Atherosclerosis, 83, 119-125.

Perkins, D. N., Pappin, D. J., Creasy, D. M., \& Cottrell, J. S. (1999). Probability-based protein identification by searching sequence databases using mass spectrometry data. Electrophoresis, 20, 3551-3567. doi:10.1002/(SICI)1522-2683(19991201)20:18<3551: AID-ELPS3551>3.0.CO;2-2.

Picotti, P., \& Aebersold, R. (2012). Selected reaction monitoringbased proteomics: Workflows, potential, pitfalls and future directions. Nature Methods, 9, 555-566. doi:10.1038/nmeth. 2015.

Porcu, P., et al. (2004). Circulating tissue kallikrein levels correlate with severity of carotid atherosclerosis. Arteriosclerosis, Thrombosis, and Vascular Biology, 24, 1104-1110. doi:10.1161/01. ATV.0000128126.57688.a9.

Posada-Ayala, M., et al. (2013). Identification of a urine metabolomic signature in patients with advanced-stage chronic kidney disease. Kidney International, . doi:10.1038/ki.2013.328.
Rein, P., Saely, C. H., Vonbank, A., Fraunberger, P., \& Drexel, H. (2014). Impact of the albumin to creatinine ratio and the coronary artery state on vascular events. American Journal of Cardiology, 113, 1616-1620. doi:10.1016/j.amjcard.2014.02. 017.

Rhee, E. P., \& Gerszten, R. E. (2012). Metabolomics and cardiovascular biomarker discovery. Clinical Chemistry, 58, 139-147. doi:10.1373/clinchem.2011.169573.

Roger, V. L. (2011). Outcomes research and epidemiology: The synergy between public health and clinical practice. Circulation: Cardiovascular Quality and Outcomes, 4, 257-259. doi:10.1161/ CIRCOUTCOMES.111.961524.

Sharma, J. N., \& Sharma, J. (2002). Cardiovascular properties of the kallikrein-kinin system. Current Medical Research and Opinion, $18,10-17$.

Shevchenko, A., Wilm, M., Vorm, O., \& Mann, M. (1996). Mass spectrometric sequencing of proteins silver-stained polyacrylamide gels. Analytical Chemistry, 68, 850-858.

Szuldrzynski, K., Zalewski, J., Machnik, A., \& Zmudka, K. (2010). Elevated levels of 8-iso-prostaglandin F2alpha in acute coronary syndromes are associated with systemic and local platelet activation. Polskie Archiwum Medycyny Wewnetrznej, 120, 19-24.

Teul, J., et al. (2009). Improving metabolite knowledge in stable atherosclerosis patients by association and correlation of GC-MS and $1 \mathrm{H}$ NMR fingerprints. Journal of Proteome Research, 8 , 5580-5589. doi:10.1021/pr900668v.

Tulpan, D., Leger, S., Belliveau, L., Culf, A., \& Cuperlovic-Culf, M. (2011). MetaboHunter: An automatic approach for identification of metabolites from 1H-NMR spectra of complex mixtures. BMC Bioinformatics, 12, 400. doi:10.1186/1471-2105-12-400.

Wagner, N. M., Bierhansl, L., Noldge-Schomburg, G., Vollmar, B., \& Roesner, J. P. (2013). Toll-like receptor 2-blocking antibodies promote angiogenesis and induce ERK1/2 and AKT signaling via CXCR4 in endothelial cells. Arteriosclerosis, Thrombosis, and Vascular Biology, 33, 1943-1951. doi:10.1161/ATVBAHA. 113.301783.

Wyss, M., \& Kaddurah-Daouk, R. (2000). Creatine and creatinine metabolism. Physiological Reviews, 80, 1107-1213.

Xia, J., Mandal, R., Sinelnikov, I. V., Broadhurst, D., \& Wishart, D. S. (2012). MetaboAnalyst 2.0-a comprehensive server for metabolomic data analysis. Nucleic Acids Research, 40, W127W133. doi:10.1093/nar/gks374.

Xia, J., Psychogios, N., Young, N., \& Wishart, D. S. (2009). MetaboAnalyst: A web server for metabolomic data analysis and interpretation. Nucleic Acids Research, 37, W652-W660. doi:10. 1093/nar/gkp356.

Yao, Y. Y., Yin, H., Shen, B., Chao, L., \& Chao, J. (2007). Tissue kallikrein infusion prevents cardiomyocyte apoptosis, inflammation and ventricular remodeling after myocardial infarction. Regulatory Peptides, 140, 12-20. doi:10.1016/j.regpep.2006.11. 020.

Yap, I. K., et al. (2010). Metabolome-wide association study identifies multiple biomarkers that discriminate north and south Chinese populations at differing risks of cardiovascular disease: INTERMAP study. Journal of Proteome Research, 9, 6647-6654. doi: $10.1021 /$ pr100798r.

Zhang, F., et al. (2009). Metabonomics study of atherosclerosis rats by ultra fast liquid chromatography coupled with ion trap-time of flight mass spectrometry. Talanta, 79, 836-844. doi:10.1016/j. talanta.2009.05.010. 